IMA Journal of Numerical Analysis (2007) 27, 151-171

doi:10.1093/imanum/drl011

Advance Access publication on May 5, 2006

\title{
A priori and a posteriori analysis of non-conforming finite elements with face penalty for advection-diffusion equations
}

\author{
L. El Alaoui $\dagger$ \\ Department of Mathematics, Imperial College, London, SW7 2AZ, UK \\ and \\ CERMICS, Ecole nationale des ponts et chaussées, Champs sur Marne, \\ 77455 Marne la Vallée Cedex 2, France \\ A. ERN† \\ CERMICS, Ecole nationale des ponts et chaussées, Champs sur Marne, \\ 77455 Marne la Vallée Cedex 2, France \\ AND \\ E. BURMAN§ \\ Institut d'Analyse et de Calcul Scientifique (CMCS/IACS), \\ Ecole Polytechnique Fédérale de Lausanne, Switzerland
}

[Received on 27 September 2005; revised on 24 March 2006]

\begin{abstract}
We analyse a non-conforming finite-element method to approximate advection-diffusion-reaction equations. The method is stabilized by penalizing the jumps of the solution and those of its advective derivative across mesh interfaces. The a priori error analysis leads to (quasi-)optimal estimates in the mesh size (sub-optimal by order $\frac{1}{2}$ in the $L^{2}$-norm and optimal in the broken graph norm for quasi-uniform meshes) keeping the Péclet number fixed. Then, we investigate a residual a posteriori error estimator for the method. The estimator is semi-robust in the sense that it yields lower and upper bounds of the error which differ by a factor equal at most to the square root of the Péclet number. Finally, to illustrate the theory we present numerical results including adaptively generated meshes.
\end{abstract}

Keywords: non-conforming finite elements; face penalty; advection; diffusion; a posteriori error estimator; adaptive meshes.

\section{Introduction}

Advection-diffusion equations in the dominant advection regime are encountered in many applications, including pollutant transport and the Navier-Stokes equations. It is well-known that the standard Galerkin approximation of these equations leads to oscillations when layers are not properly resolved. To stabilize this phenomenon, several well-established techniques have been proposed and analysed in a conforming setting (e.g. streamline-diffusion, Brooks \& Hughes, 1982; Johnson et al., 1984, subgrid

\footnotetext{
$\dagger$ Corresponding author. Email: 1.elalaoui@imperial.ac.uk

\$Email: ern@cermics.enpc.fr

§Email: erik.burman@epfl.ch
} 
viscosity, Guermond, 1999, 2001, and residual free bubbles, Brezzi \& Russo, 1994) as well as in a discontinuous setting (e.g. the discontinuous Galerkin method in Johnson \& Pitkäranta, 1986).

An interesting compromise between conforming and discontinuous Galerkin methods consists of using non-conforming finite elements. In this paper, we are interested in low-order non-conforming finite elements such as the Crouzeix-Raviart finite element. This finite element presents various interesting features. First, the degrees of freedom are localized at the mesh faces, thereby leading to efficient communication and parallelization. Second, Crouzeix-Raviart finite elements have close links with finitevolume box schemes; see, e.g. Courbet \& Croisille (1998) and Croisille (2000) for Darcy's equations and El Alaoui \& Ern (2006) for advection-diffusion equations. This property is useful to reconstruct locally the diffusive flux in problems where conservativity properties are important, e.g. pollutant transport. Finally, keeping the mesh fixed, the Crouzeix-Raviart finite-element space has approximately twice fewer degrees of freedom than the first-order discontinuous Galerkin finite-element space.

The topic of approximating advection-diffusion equations by Crouzeix-Raviart finite elements is not new; see John et al. (1998) and Matthies \& Tobiska (2001) where the streamline-diffusion paradigm is extended to the non-conforming setting. The difficulties with streamline-diffusion, in both conforming and non-conforming settings, are that the method involves a parameter depending on the diffusion coefficient and that the extension to time-dependent problems is not straightforward. This can be impractical in non-linear problems, e.g. the Navier-Stokes equations where the regions with dominant convection may not be known a priori. In this paper, we consider a different technique to stabilize the non-conforming finite-element approximation, namely that of penalizing the jumps of the solution and those of its advective derivative across mesh interfaces. Drawing on earlier ideas by Douglas \& Dupont (1976), the analysis of face penalty finite-element methods has been recently extended to advection-diffusion equations with dominant advection (Burman, 2005; Burman \& Hansbo, 2006) and to the Stokes equations (Burman \& Hansbo, 2004); see also Ouazzi \& Turek (2005) for an application to incompressible flow problems. The advantage of using the face penalty technique rather than streamline-diffusion is that the former involves a single user-dependent parameter which is independent of the diffusion coefficient. Moreover, the face penalty technique is readily extendable to time-dependent problems.

The a posteriori error analysis of non-conforming finite-element approximations to advectiondiffusion equations is a much less explored topic. Even in a conforming setting, the analysis is harder than it seems at the first sight. The main issue at stake is to derive a so-called robust error estimator for which the upper and lower bounds for the error differ by a factor that is independent of the Péclet number. The first main advance in this direction was achieved by Verfürth (1998) in a conforming setting, the proposed error estimator yielding a factor between lower and upper error bounds which scales at most as the square root of the Péclet number. Such error estimators are henceforth called semi-robust. Further results in this direction include Araya et al. (2005a,b) and Berrone (2002). Recently, robust error estimators, still in a conforming setting, have been proposed by Verfürth (2005) and Sangalli (2004b). For this purpose, the norm in which the error is measured has to be modified; in particular, it includes the advective derivative of the error. In Verfürth (2005), the advective derivative is measured in a dual (non-local) norm. In Sangalli (2004b), the advective derivative is measured in a non-standard interpolated norm of order $\frac{1}{2}$ introduced in Sangalli (2004a) and which can be evaluated by solving a generalized eigenvalue problem on a fine mesh. The purpose of the present work is to propose and analyse a semi-robust error estimator for non-conforming finite-element approximations to advection-diffusion equations. To our knowledge, it is the first semi-robust error estimator in this setting. The present analysis can be viewed as a first step towards establishing robust error estimators in the non-conforming setting. 
This paper is organized as follows. Section 2 presents the model problem and the non-conforming finite-element approximation with face penalty. Section 3 deals with the a priori error analysis and Section 4 with the residual a posteriori error analysis. Section 5 contains numerical results and Section 6 draws the conclusions.

\section{The setting}

\subsection{The model problem}

Let $\Omega$ be a polygonal domain of $\mathbb{R}^{d}$ with Lipschitz boundary $\partial \Omega$ and outward normal $n$. Let $\varepsilon>0$, $\beta \in\left[\mathscr{C}^{0, \frac{1}{2}}(\bar{\Omega})\right]^{d}$ and $\theta \in L^{\infty}(\Omega)$ be, respectively, the diffusion coefficient, the velocity field and the reaction coefficient. Set $\partial \Omega_{\text {in }}=\{x \in \partial \Omega: \beta \cdot n<0\}$ and $\partial \Omega_{\text {out }}=\{x \in \partial \Omega: \beta \cdot n \geqslant 0\}$. Let $f \in L^{2}(\Omega)$ and $g \in L^{2}\left(\partial \Omega_{\text {in }}\right)$ be the data. We are interested in the following advection-diffusion-reaction problem with mixed Robin-Neumann boundary conditions:

$$
\begin{cases}-\varepsilon \Delta u+\beta \cdot \nabla u+\theta u=f & \text { in } \Omega, \\ -\varepsilon \nabla u \cdot n+\beta \cdot n u=g & \text { on } \partial \Omega_{\text {in }} \\ \nabla u \cdot n=0 & \text { on } \partial \Omega_{\text {out }}\end{cases}
$$

Without loss of generality, we assume that (2.1) is non-dimensionalized so that $\|\beta\|_{\left[L^{\infty}(\Omega)\right]^{d}}$ and the length scale of $\Omega$ are of order unity; hence, the parameter $\varepsilon$ is the reciprocal of the Péclet number.

Under the assumption that there is $\sigma_{0}>0$ such that $\sigma=\theta-\frac{1}{2} \nabla \cdot \beta \geqslant \sigma_{0}$ in $\Omega$ and that $\nabla \cdot \beta \in$ $L^{\infty}(\Omega)$, it is straightforward to verify using the Lax-Milgram Lemma that the following weak formulation of (2.1) is well-posed:

$$
\left\{\begin{array}{l}
\text { seek } u \in H^{1}(\Omega) \text { such that } \\
a(u, v)=\int_{\Omega} f v-\int_{\partial \Omega_{\text {in }}} g v \quad \forall v \in H^{1}(\Omega),
\end{array}\right.
$$

where

$$
a(u, v)=\int_{\Omega} \varepsilon \nabla u \cdot \nabla v+\int_{\Omega}(\theta-\nabla \cdot \beta) u v-\int_{\Omega} u(\beta \cdot \nabla v)+\int_{\partial \Omega_{\mathrm{out}}}(\beta \cdot n) u v .
$$

\subsection{The discrete setting}

Let $\left(\mathscr{T}_{h}\right)_{h}$ be a shape-regular family of simplicial affine meshes of $\Omega$. For an element $T \in \mathscr{T}_{h}$, let $\partial T$ denote its boundary, $h_{T}$ its diameter and set $h=\max _{T \in \mathscr{T}_{h}} h_{T}$. Henceforth, the notation $\xi \lesssim \zeta$ means that there is a positive $c$, independent of any mesh size and of $\varepsilon$, such that $\xi \leqslant c \zeta$. Since the advectiondiffusion problem has been non-dimensionalized so that the field $\beta$ is of order unity, the dependency on $\beta$ can be hidden in the constants in the error estimates. The same is done for the function $\theta$ since we are not interested in the asymptotics of strong reaction regimes.

Let $\mathscr{F}_{h}, \mathscr{F}_{h}^{i}$ and $\mathscr{F}_{h}^{\partial}$ denote, respectively, the set of faces, internal and external faces in $\mathscr{T}_{h}$. Let $\mathscr{F}_{h}^{\text {in }}$ and $\mathscr{F}_{h}{ }_{h}$ be the set of faces belonging, respectively, to $\partial \Omega_{\text {in }}$ and $\partial \Omega_{\text {out }}$ so that $\mathscr{F}_{h}^{\partial}=\mathscr{F}_{h}^{\text {in }} \cup \mathscr{F}_{h}$ out . For a face $F \in \mathscr{F}_{h}$, let $h_{F}$ denote its diameter and $\mathscr{T}_{F}$ the set of elements in $\mathscr{T}_{h}$ containing $F$. For an element $T \in \mathscr{T}_{h}$, let $\mathscr{F}_{T}$ denote the set of faces belonging to $T$. Let $\mathscr{S}_{h}$ be the set of mesh vertices. For a vertex $s \in \mathscr{S}_{h}$, let $\mathscr{T}_{s}$ denote the set of elements in $\mathscr{T}_{h}$ containing $s$. 
For an integer $k \geqslant 1$, let $H^{k}\left(\mathscr{T}_{h}\right)=\left\{v \in L^{2}(\Omega): \forall T \in \mathscr{T}_{h},\left.v\right|_{T} \in H^{k}(T)\right\}$. We introduce the discrete gradient operator $\nabla_{h}: H^{1}\left(\mathscr{T}_{h}\right) \rightarrow\left[L^{2}(\Omega)\right]^{d}$ such that for all $v \in H^{1}\left(\mathscr{T}_{h}\right)$ and for all $T \in$ $\mathscr{T}_{h},\left.\left(\nabla_{h} v\right)\right|_{T}=\nabla\left(\left.v\right|_{T}\right)$. Let $F \in \mathscr{F}_{h}^{i}$; then, there are $T_{1}(F)$ and $T_{2}(F) \in \mathscr{T}_{h}$ such that $F=T_{1}(F) \cap$ $T_{2}(F)$. Conventionally, choose $n_{F}$ to be the unit normal vector to $F$ pointing from $T_{1}(F)$ towards $T_{2}(F)$. For $v \in H^{1}\left(\mathscr{T}_{h}\right)$, define its jump across $F$ as

$$
\llbracket v \rrbracket_{F}=\left.v\right|_{T_{1}(F)}-\left.v\right|_{T_{2}(F)} \quad \text { a.e. on } F .
$$

For $F \in \mathscr{F}_{h}^{\partial}$, define $n_{F}$ to be the unit normal to $F$ pointing towards the exterior of $\Omega$ and for $v \in$ $H^{1}\left(\mathscr{T}_{h}\right)$, set $\llbracket v \rrbracket_{F}=\left.v\right|_{T(F)}$ where $T(F)$ is the mesh element of which $F$ is a face. A similar notation is used for the jumps of vector-valued functions, the jump being taken componentwise.

For a measurable subset $R \subset \Omega,(\cdot, \cdot)_{0, R}$ denotes the $L^{2}(R)$-scalar product, $\|\cdot\|_{0, R}$ the associated norm, $\|\cdot\|_{k, R}$ the $H^{k}(R)$-norm for $k \geqslant 1$ and $\beta_{\infty, R}$ the $\left[L^{\infty}(R)\right]^{d}$-norm of the function $\beta$.

Consider the Crouzeix-Raviart finite-element space $P_{\mathrm{nc}}^{1}\left(\mathscr{T}_{h}\right)$ defined as (Crouzeix \& Raviart, 1973)

$$
P_{\mathrm{nc}}^{1}\left(\mathscr{T}_{h}\right)=\left\{v_{h} \in L^{2}(\Omega): \forall T \in \mathscr{T}_{h},\left.v_{h}\right|_{T} \in P^{1}(T) \forall F \in \mathscr{F}_{h}^{i}, \int_{F} \llbracket v_{h} \rrbracket_{F}=0\right\},
$$

where $P^{1}(T)$ denotes the vector space of polynomials on $T$ with degree less than or equal to 1 . For further purposes, we restate some well-known results. For all $v_{h} \in P_{\mathrm{nc}}^{1}\left(\mathscr{T}_{h}\right)$, for all $T \in \mathscr{T}_{h}$ and for all $F \subset \partial T$,

$$
\begin{aligned}
\left\|v_{h}\right\|_{1, T} & \lesssim h_{T}^{-1}\left\|v_{h}\right\|_{0, T}, \\
\left\|v_{h}\right\|_{0, F} & \lesssim h_{F}^{-\frac{1}{2}}\left\|v_{h}\right\|_{0, T}, \\
\left\|\llbracket v_{h} \rrbracket_{F}\right\|_{0, F} & \lesssim h_{F}\left\|\llbracket \nabla_{h} v_{h} \rrbracket_{F}\right\|_{0, F} .
\end{aligned}
$$

Let $P_{\mathrm{c}}^{1}\left(\mathscr{T}_{h}\right)=P_{\mathrm{nc}}^{1}\left(\mathscr{T}_{h}\right) \cap H^{1}(\Omega)$ be the usual first-order conforming finite-element space. Let $\mathscr{I}_{\text {Os }}$ : $P_{\text {nc }}^{1}\left(\mathscr{T}_{h}\right) \rightarrow P_{\mathrm{c}}^{1}\left(\mathscr{T}_{h}\right)$ be the so-called Oswald interpolation operator (El Alaoui \& Ern, 2004; Hoppe \& Wohlmuth, 1996) defined as follows:

$$
\forall v_{h} \in P_{\mathrm{nc}}^{1}\left(\mathscr{T}_{h}\right), \quad \forall s \in \mathscr{S}_{h}, \quad \mathscr{I}_{\mathrm{Os}} v_{h}(s)=\left.\frac{1}{\sharp\left(\mathscr{T}_{s}\right)} \sum_{T \in \mathscr{T}_{s}} v_{h}\right|_{T}(s),
$$

where $\sharp\left(\mathscr{T}_{s}\right)$ denotes the cardinal number of $\mathscr{T}_{s}$. This operator is endowed with the following approximation property (Achdou et al., 2003; Burman, 2005; Karakashian \& Pascal, 2003): for all $v_{h} \in P_{\mathrm{nc}}^{1}\left(\mathscr{T}_{h}\right)$ and for all $T \in \mathscr{T}_{h}$,

$$
\left\|v_{h}-\mathscr{I}_{\mathrm{Os}} v_{h}\right\|_{0, T}+h_{T}\left\|\nabla\left(v_{h}-\mathscr{I}_{\mathrm{Os}} v_{h}\right)\right\|_{0, T} \lesssim \sum_{F \in \mathscr{F}_{T}^{\mathrm{Os}}} h_{F}^{\frac{1}{2}}\left\|\llbracket v_{h} \rrbracket_{F}\right\|_{0, F},
$$

where $\mathscr{F}_{T}^{\mathrm{Os}}$ denotes all the interior faces in the mesh containing a vertex of $T$. Using (2.7) and (2.9) yields

$$
\left\|v_{h}-\mathscr{I}_{\mathrm{Os}} v_{h}\right\|_{0, T}+h_{T}\left\|\nabla\left(v_{h}-\mathscr{I}_{\mathrm{Os}} v_{h}\right)\right\|_{0, T} \lesssim \sum_{F \in \mathscr{F}_{T}^{\mathrm{Os}}} h_{F}^{\frac{3}{2}}\left\|\llbracket \nabla_{h} v_{h} \rrbracket_{F}\right\|_{0, F} .
$$




\subsection{The discrete bilinear forms}

Set $V=H^{2}\left(\mathscr{T}_{h}\right) \cap H^{1}(\Omega)$ and $V(h)=V+P_{\mathrm{nc}}^{1}\left(\mathscr{T}_{h}\right)$. Introduce the bilinear form $a_{h}$ defined on $V(h) \times V(h)$ by

$$
\begin{aligned}
a_{h}(v, w)= & \int_{\Omega} \varepsilon \nabla_{h} v \cdot \nabla_{h} w+\int_{\Omega}(\theta-\nabla \cdot \beta) v w-\int_{\Omega} v\left(\beta \cdot \nabla_{h} w\right) \\
& +\sum_{F \in \mathscr{F}_{h}^{i}} \int_{F} \beta \cdot n_{F} \llbracket v w \rrbracket_{F}+\int_{\partial \Omega_{\mathrm{out}}}(\beta \cdot n) v w
\end{aligned}
$$

and equip $V(h)$ with the norm

$$
\|v\|_{\varepsilon \beta \sigma, \Omega}=\left\|\varepsilon^{\frac{1}{2}} \nabla_{h} v\right\|_{0, \Omega}+\left\|\sigma^{\frac{1}{2}} v\right\|_{0, \Omega}+\left\||\beta \cdot n|^{\frac{1}{2}} v\right\|_{0, \partial \Omega} .
$$

The bilinear form $a_{h}$ is not $\|\cdot\|_{\varepsilon \beta \sigma, \Omega}$-coercive on $V(h)$ due to the presence of the jump terms in (2.11). To control these terms, consider the bilinear form $j_{h}$ defined on $V(h) \times V(h)$ by

$$
j_{h}(v, w)=\sum_{F \in \mathscr{F}_{h}^{i}} \int_{F}\left(\beta \cdot n_{F}\right) \llbracket v \rrbracket_{F} w^{\downarrow},
$$

where $w^{\downarrow}$ is the so-called downwind value of $w$ defined as $w^{\downarrow}=\left.w\right|_{T_{2}(F)}$ if $\beta \cdot n_{F} \geqslant 0$ and $w^{\downarrow}=\left.w\right|_{T_{1}(F)}$ otherwise.

LEMMA 2.1 For all $v \in V(h)$,

$$
\|v\|_{\varepsilon \beta \sigma, \Omega}^{2}+\sum_{F \in \mathscr{F}_{h}^{i}}\left\|\left|\beta \cdot n_{F}\right|^{\frac{1}{2}} \llbracket v \rrbracket_{F}\right\|_{0, F}^{2} \lesssim a_{h}(v, v)+j_{h}(v, v) .
$$

Proof. Straightforward verification using integration by parts.

Working with the bilinear form $a_{h}+j_{h}$ alone is not sufficient to control the advective derivative of the discrete solution. For this purpose, we introduce the bilinear form $s_{h}$ on $V(h) \times V(h)$ such that

$$
s_{h}(v, w)=\sum_{F \in \mathscr{F}_{h}^{i}} \int_{F} \gamma \frac{h_{F}^{2}}{\beta_{\infty, F}} \llbracket \beta \cdot \nabla_{h} v \rrbracket_{F} \llbracket \beta \cdot \nabla_{h} w \rrbracket_{F},
$$

where $\gamma>0$ is independent of any mesh size and of $\varepsilon$ (the contribution of a face $F \in \mathscr{F}_{h}^{i}$ is conventionally set to zero if $\beta_{\infty, F}=0$ ). This leads to the following discrete problem:

$$
\left\{\begin{array}{l}
\text { seek } u_{h} \in P_{\mathrm{nc}}^{1}\left(\mathscr{T}_{h}\right) \text { such that for all } v_{h} \in P_{\mathrm{nc}}^{1}\left(\mathscr{T}_{h}\right), \\
a_{h}\left(u_{h}, v_{h}\right)+j_{h}\left(u_{h}, v_{h}\right)+s_{h}\left(u_{h}, v_{h}\right)=\left(f, v_{h}\right)_{0, \Omega}-\left(g, v_{h}\right)_{0, \partial \Omega_{\mathrm{in}}}
\end{array}\right.
$$

Lemma 2.1 implies that the bilinear form $\left(a_{h}+j_{h}+s_{h}\right)$ is $\|\cdot\|_{\varepsilon \beta \sigma, \Omega}$-coercive; hence, (2.16) is well-posed owing to the Lax-Milgram Lemma.

REMARK 2.1 A term similar to the bilinear form $j_{h}$ is also added in John et al. (1998) to control the jumps across mesh interfaces. As in the discrete problem (2.16) where the bilinear form $j_{h}$ is 
introduced in addition to the bilinear form $s_{h}$, this term is introduced in addition to the streamlinediffusion term stabilizing the non-conforming finite-element approximation. To avoid this additional term, it is possible to work with the $Q_{\text {rot }}^{1}$ finite element on rectangular meshes (Stynes \& Tobiska, 2001) or to consider a non-conforming finite-element space satisfying the patch-test of order three (Knobloch \& Tobiska, 2003); however, the dimension of this space is twice as large as the dimension of the CrouzeixRaviart finite-element space. Alternatively, one can penalize the jumps of all the gradient components instead of just those of the advective components and take $j_{h}=0$; we refer to Burman (2005) for more details.

\section{A priori error analysis}

In this section, we present the convergence analysis for the discrete problem (2.16). The main result is Theorem 3.1. Without loss of generality, we assume that $h \leqslant 1$ and $\varepsilon \leqslant 1$.

The error analysis is performed in the spirit of the second Strang Lemma by considering two norms on $V(h)$, namely,

$$
\begin{gathered}
\|w\|_{A, \Omega}=\|w\|_{\varepsilon \beta \sigma, \Omega}+\left(\sum_{F \in \mathscr{F}_{h}^{i}}\left\|\left|\beta \cdot n_{F}\right|^{\frac{1}{2}} \llbracket w \rrbracket_{F}\right\|_{0, F}^{2}\right)^{\frac{1}{2}}+s_{h}(w, w)^{\frac{1}{2}}, \\
\|w\|_{h, \frac{1}{2}}=\|w\|_{A, \Omega}+\left(\sum_{T \in \mathscr{T}_{h}} h_{T}^{-1}\|w\|_{0, T}^{2}+\|w\|_{0, \partial T}^{2}\right)^{\frac{1}{2}} .
\end{gathered}
$$

Let $u$ be the unique solution to (2.2) and let $u_{h}$ be the unique solution to (2.16).

LEMMA 3.1 (Stability) The bilinear form $\left(a_{h}+j_{h}+s_{h}\right)$ is $\|\cdot\|_{A, \Omega}$-coercive.

Proof. Direct consequence of Lemma 2.1.

LEMMA 3.2 (Continuity) Let $\Pi_{h}$ be the $L^{2}$-orthogonal projection onto $P_{\mathrm{c}}^{1}\left(\mathscr{T}_{h}\right)$. Then, for all $w \in V$ and for all $w_{h} \in P_{\mathrm{nc}}^{1}\left(\mathscr{T}_{h}\right)$,

$$
a_{h}\left(\Pi_{h} w-w, w_{h}\right) \lesssim\left\|\Pi_{h} w-w\right\|_{h, \frac{1}{2}}\left\|w_{h}\right\|_{A, \Omega} .
$$

Proof. Let $w \in V$ and set $v=\Pi_{h} w-w$. Let $w_{h} \in P_{\mathrm{nc}}^{1}\left(\mathscr{T}_{h}\right)$ and let us estimate each term in $a_{h}\left(v, w_{h}\right)$.

(1) It is clear that

$$
\int_{\Omega} \varepsilon \nabla_{h} v \cdot \nabla_{h} w_{h}+\int_{\Omega}(\theta-\nabla \cdot \beta) v w_{h} \lesssim\|v\|_{\varepsilon \beta \sigma, \Omega}\left\|w_{h}\right\|_{\varepsilon \beta \sigma, \Omega} \lesssim\|v\|_{h, \frac{1}{2}}\left\|w_{h}\right\|_{A, \Omega} .
$$

(2) Let us write $\int_{\Omega} v \beta \cdot \nabla_{h} w_{h}=\int_{\Omega} v\left(\beta-\beta_{h}^{1}\right) \cdot \nabla_{h} w_{h}+\int_{\Omega} v \beta_{h}^{1} \cdot \nabla_{h} w_{h}$ where $\beta_{h}^{1}$ is the $L^{2}$-orthogonal projection of $\beta$ onto $P^{1}\left(\mathscr{T}_{h}\right):=\left\{v_{h} \in L^{2}(\Omega): \forall T \in \mathscr{T}_{h},\left.v_{h}\right|_{T} \in P^{1}(T)\right\}$. Since $\beta \in$ $\left[\mathscr{C}^{0, \frac{1}{2}}(\Omega)\right]^{d}$ and owing to the inverse inequality (2.5),

$$
\int_{\Omega} v\left(\beta-\beta_{h}^{1}\right) \cdot \nabla_{h} w_{h} \lesssim \sum_{T \in \mathscr{T}_{h}} h_{T}^{-\frac{1}{2}}\|v\|_{0, T}\left\|w_{h}\right\|_{0, T} \lesssim\|v\|_{h, \frac{1}{2}}\left\|w_{h}\right\|_{A, \Omega} .
$$


Furthermore, by construction, $\left(v, \mathscr{I}_{\mathrm{Os}}\left(\beta_{h}^{1} \cdot \nabla_{h} w_{h}\right)\right)_{0, \Omega}=0$; hence, using (2.5), (2.6), (2.10), the regularity of $\beta$ and the shape-regularity of the mesh family yields

$$
\begin{aligned}
\int_{\Omega} v \beta_{h}^{1} \cdot \nabla_{h} w_{h}= & \int_{\Omega} v\left(\beta_{h}^{1} \cdot \nabla_{h} w_{h}-\mathscr{I}_{\mathrm{Os}}\left(\beta_{h}^{1} \cdot \nabla_{h} w_{h}\right)\right) \\
\lesssim & \sum_{T \in \mathscr{T}_{h}}\|v\|_{0, T}\left(\sum_{F \in \mathscr{F}_{T}^{\mathrm{Os}}} h_{F}^{\frac{1}{2}}\left\|\llbracket \beta_{h}^{1} \cdot \nabla_{h} w_{h} \rrbracket_{F}\right\|_{0, F}\right) \\
\lesssim & \sum_{T \in \mathscr{T}_{h}}\|v\|_{0, T}\left(\sum_{F \in \mathscr{F}_{T}^{\mathrm{Os}}} h_{F}^{\frac{1}{2}}\left\|\llbracket \beta \cdot \nabla_{h} w_{h} \rrbracket_{F}\right\|_{0, F}\right) \\
& +\sum_{T \in \mathscr{T}_{h}}\|v\|_{0, T}\left(\sum_{F \in \mathscr{F}_{T}^{\mathrm{Os}}} h_{F}^{\frac{1}{2}}\left\|\llbracket\left(\beta_{h}^{1}-\beta\right) \cdot \nabla_{h} w_{h} \rrbracket_{F}\right\|_{0, F}\right) \\
\lesssim & \left(\sum_{T \in \mathscr{T}_{h}} h_{T}^{-1}\|v\|_{0, T}^{2}\right)^{\frac{1}{2}}\left(s_{h}\left(w_{h}, w_{h}\right)^{\frac{1}{2}}+\left\|w_{h}\right\|_{0, \Omega}\right) \lesssim\|v\|_{h, \frac{1}{2}}\left\|w_{h}\right\|_{A, \Omega} .
\end{aligned}
$$

(3) Since $v \in H^{1}(\Omega), \beta \cdot n_{F} \llbracket v w_{h} \rrbracket_{F}=\beta \cdot n_{F} v \llbracket w_{h} \rrbracket_{F}$. Hence,

$$
\begin{aligned}
& \sum_{F \in \mathscr{F}_{h}^{i}} \int_{F} \beta \cdot n_{F} \llbracket v w_{h} \rrbracket_{F} \lesssim\left(\sum_{T \in \mathscr{T}_{h}}\|v\|_{0, \partial T}^{2}\right)^{\frac{1}{2}}\left(\sum_{F \in \mathscr{F}_{h}^{i}}\left\|\left|\beta \cdot n_{F}\right|^{\frac{1}{2}} \llbracket w_{h} \rrbracket_{F}\right\|_{0, F}^{2}\right)^{\frac{1}{2}} \\
& \lesssim\|v\|_{h, \frac{1}{2}}\left\|w_{h}\right\|_{A, \Omega} .
\end{aligned}
$$

Similarly,

$$
\sum_{F \in \mathscr{F}_{h}^{\text {out }}} \int_{F}(\beta \cdot n) v w_{h} \lesssim\|v\|_{h, \frac{1}{2}}\left\|w_{h}\right\|_{A, \Omega} .
$$

Collecting the above inequalities yields (3.3).

LEMMA 3.3 (Error estimation) Assume that $u \in H^{2}(\Omega)$. Set

$$
R_{h}(u)=\sup _{w_{h} \in P_{\mathrm{nc}}^{1}\left(\mathscr{T}_{h}\right)} \frac{a_{h}\left(u, w_{h}\right)-\left(f, w_{h}\right)_{0, \Omega}+\left(g, w_{h}\right)_{0, \partial} \Omega_{\mathrm{in}}}{\left\|w_{h}\right\|_{A, \Omega}} .
$$

Then,

$$
\left\|u-u_{h}\right\|_{A, \Omega} \lesssim\left\|u-\Pi_{h} u\right\|_{h, \frac{1}{2}}+R_{h}(u) .
$$


Proof. Since $\left\|u-\Pi_{h} u\right\|_{A, \Omega} \leqslant\left\|u-\Pi_{h} u\right\|_{h, \frac{1}{2}}$, the triangle inequality yields

$$
\left\|u-u_{h}\right\|_{A, \Omega} \leqslant\left\|u-\Pi_{h} u\right\|_{h, \frac{1}{2}}+\left\|\Pi_{h} u-u_{h}\right\|_{A, \Omega} .
$$

Set $w_{h}=\Pi_{h} u-u_{h}$ and observe that $w_{h} \in V(h)$. Then, the $\|\cdot\|_{A, \Omega}$-coercivity of $\left(a_{h}+j_{h}+s_{h}\right)$ on $V(h) \times V(h)$ yields

$$
\left\|\Pi_{h} u-u_{h}\right\|_{A, \Omega}^{2} \lesssim a_{h}\left(\Pi_{h} u-u_{h}, w_{h}\right)+j_{h}\left(\Pi_{h} u-u_{h}, w_{h}\right)+s_{h}\left(\Pi_{h} u-u_{h}, w_{h}\right) .
$$

Moreover, using the fact that $s_{h}\left(u, w_{h}\right)=j_{h}\left(\Pi_{h} u, w_{h}\right)=0$ since $u \in H^{2}(\Omega)$ and $\Pi_{h} u \in H^{1}(\Omega)$ leads to

$$
\begin{aligned}
& a_{h}\left(\Pi_{h} u-u_{h}, w_{h}\right)+j_{h}\left(\Pi_{h} u-u_{h}, w_{h}\right)+s_{h}\left(\Pi_{h} u-u_{h}, w_{h}\right) \\
& \quad=a_{h}\left(\Pi_{h} u-u, w_{h}\right)+s_{h}\left(\Pi_{h} u-u, w_{h}\right)+a_{h}\left(u, w_{h}\right)-\left(f, w_{h}\right)_{0, \Omega}+\left(g, w_{h}\right)_{0, \partial} \Omega_{\text {in }} .
\end{aligned}
$$

Owing to Lemma 3.2,

$$
a_{h}\left(\Pi_{h} u-u, w_{h}\right) \lesssim\left\|\Pi_{h} u-u\right\|_{h, \frac{1}{2}}\left\|w_{h}\right\|_{A, \Omega}
$$

Furthermore,

$$
s_{h}\left(\Pi_{h} u-u, w_{h}\right) \leqslant s_{h}\left(\Pi_{h} u-u, \Pi_{h} u-u\right)^{\frac{1}{2}} s_{h}\left(w_{h}, w_{h}\right)^{\frac{1}{2}} \leqslant\left\|\Pi_{h} u-u\right\|_{h, \frac{1}{2}}\left\|w_{h}\right\|_{A, \Omega} .
$$

The conclusion is straightforward.

Lemma 3.4 (Consistency) Assume that $u \in H^{2}(\Omega)$. Then,

$$
\left|R_{h}(u)\right| \lesssim \varepsilon^{\frac{1}{2}} h\|u\|_{2, \Omega} .
$$

Proof. Let $w_{h} \in P_{\mathrm{nc}}^{1}\left(\mathscr{T}_{h}\right)$. Observe that

$$
a_{h}\left(u, w_{h}\right)-\left(f, w_{h}\right)_{0, \Omega}+\left(g, w_{h}\right)_{0, \partial \Omega_{\mathrm{in}}}=\sum_{F \in \mathscr{F}_{h}^{i}} \int_{F} \varepsilon \nabla u \cdot n_{F} \llbracket w_{h} \rrbracket_{F} .
$$

Since $P_{\mathrm{nc}}^{1}\left(\mathscr{T}_{h}\right)$ satisfies the patch-test of order zero,

$$
a_{h}\left(u, w_{h}\right)-\left(f, w_{h}\right)_{\Omega}+\left(g, w_{h}\right)_{0, \partial} \Omega_{\text {in }}=\sum_{F \in \mathscr{F}_{h}^{i}} \int_{F} \varepsilon\left(\nabla u-\Pi_{F}^{0}(\nabla u)\right) \cdot n_{F} \llbracket w_{h} \rrbracket_{F},
$$

where $\Pi_{F}^{0}:\left[L^{2}(F)\right]^{d} \rightarrow\left[P^{0}(F)\right]^{d}$ denotes the $L^{2}$-orthogonal projection on $\left[P^{0}(F)\right]^{d}$. Using the approximation properties of the $L^{2}$-orthogonal projection onto $\left[P^{0}(F)\right]^{d}$ and proceeding as in Crouzeix \& Raviart (1973) yields

$$
\left|a_{h}\left(u, w_{h}\right)-\left(f, w_{h}\right)_{\Omega}+\left(g, w_{h}\right)_{\partial \Omega_{\text {in }}}\right| \lesssim \varepsilon h\|u\|_{2, \Omega}\left\|\nabla_{h} w_{h}\right\|_{0, \Omega},
$$

whence (3.6) is readily inferred. 
THEOREM 3.1 (Convergence) Assume that $u \in H^{2}(\Omega)$. Then,

$$
\left\|u-u_{h}\right\|_{A, \Omega} \lesssim h\left(\varepsilon^{\frac{1}{2}}+h^{\frac{1}{2}}\right)\|u\|_{2, \Omega} .
$$

Proof. Observe that $\Pi_{h}$ satisfies the following approximation property (see, e.g. Boman (2000) for local approximation properties of $\Pi_{h}$ ): for all $v \in H^{2}(\Omega)$,

$$
\left\|v-\Pi_{h} v\right\|_{h, \frac{1}{2}} \lesssim h\left(\varepsilon^{\frac{1}{2}}+h^{\frac{1}{2}}\right)\|v\|_{2, \Omega},
$$

and use Lemmata 3.3 and 3.4.

REMARK 3.1 The a priori error estimate (3.7) shows that when keeping the Péclet number $\varepsilon^{-1}$ fixed, the convergence order in the mesh size for the error $\left\|u-u_{h}\right\|_{A, \Omega}$ is 1 in the diffusion-dominated regime and $\frac{3}{2}$ in the advection-dominated regime. This estimate is similar to those derived for stabilized schemes in the conforming setting; see, e.g. Brezzi \& Russo (1994), Brooks \& Hughes (1982), Burman \& Hansbo (2004) and Guermond (1999).

REMARK 3.2 The above analysis shares some common features with those presented in Burman (2005). The main differences are that we consider mixed Robin-Neumann boundary conditions instead of Dirichlet boundary conditions, that the advective field is in $\left[\mathscr{C}^{0, \frac{1}{2}}(\Omega)\right]^{d}$ instead of being piecewise affine and that the stabilization is achieved by using the bilinear form $\left(j_{h}+s_{h}\right)$ instead of penalizing the jumps of all the gradient components across interfaces.

\section{A posteriori error analysis}

In this section, we present the residual a posteriori error analysis for the discrete problem (2.16). The main results are Theorem 4.1 which yields an upper bound for the error and Theorem 4.2 which yields a lower bound for the error.

Let $f_{h}, g_{h}, \beta_{h}$ and $\theta_{h}$ be, respectively, the $L^{2}$-orthogonal projection of $f, g, \beta$ and $\theta$ onto the space of (discontinuous) piecewise polynomials of degree at most 1. Let $u$ be the unique solution to (2.2) and let $u_{h}$ be the unique solution to (2.16). As in Section 3, we assume without loss of generality that $h \leqslant 1$ and $\varepsilon \leqslant 1$. Furthermore, define

$$
\alpha_{S}=\min \left(\varepsilon^{-\frac{1}{2}} h_{S}, 1\right)
$$

where $S$ belongs to $\mathscr{T}_{h}$ or $\mathscr{F}_{h}$, and observe that

$$
\begin{gathered}
h_{S}^{\frac{1}{2}} \leqslant \max \left(\varepsilon^{\frac{1}{2}}, \alpha_{S}\right), \\
h_{S} \leqslant \alpha_{S} .
\end{gathered}
$$

Indeed, (4.2) trivially holds if $h_{S} \leqslant \varepsilon$ whereas if $\varepsilon \leqslant h_{S}$, then $h_{S}^{\frac{1}{2}} \leqslant \alpha_{S}$. Furthermore, (4.3) directly results from the fact that $h \leqslant 1$ and $\varepsilon \leqslant 1$.

THEOREM 4.1 (Upper bound) The following holds:

$$
\left\|u-u_{h}\right\|_{\varepsilon \beta \sigma, \Omega} \lesssim\left(\sum_{T \in \mathscr{T}_{h}}\left[\eta_{T}\left(u_{h}\right)^{2}+\delta_{T}\left(u_{h}\right)^{2}\right]+\sum_{F \in \mathscr{F}_{h}^{i}} \eta_{F}\left(u_{h}\right)^{2}\right)^{\frac{1}{2}},
$$


where we have introduced for all $T \in \mathscr{T}_{h}$ the local data error indicators

$$
\begin{aligned}
\delta_{T}\left(u_{h}\right)= & \alpha_{T}\left(\left\|f-f_{h}\right\|_{0, T}+\left\|\left(\beta-\beta_{h}\right) \cdot \nabla u_{h}\right\|_{0, T}+\left\|\left(\theta-\theta_{h}\right) u_{h}\right\|_{0, T}\right) \\
& +\sum_{F \in \mathscr{F}_{T}^{(2)}} \varepsilon^{-\frac{1}{4}} \alpha_{F}^{\frac{1}{2}}\left\|g-g_{h}+\left(\beta-\beta_{h}\right) \cdot n_{F} u_{h}\right\|_{0, F},
\end{aligned}
$$

as well as the local residual error indicators

$$
\begin{gathered}
\eta_{T}\left(u_{h}\right)=\alpha_{T}\left\|f_{h}-\beta_{h} \cdot \nabla u_{h}-\theta_{h} u_{h}\right\|_{0, T}+\sum_{F \in \mathscr{F}_{T}^{(1)}} \varepsilon^{-\frac{1}{4}} \alpha_{F}^{\frac{1}{2}}\left\|\varepsilon \llbracket \nabla \nabla_{h} u_{h} \rrbracket_{F} \cdot n_{F}\right\|_{0, F} \\
+\sum_{F \in \mathscr{F}_{T}^{(2)}} \varepsilon^{-\frac{1}{4}} \alpha_{F}^{\frac{1}{2}}\left\|g_{h}+\varepsilon \nabla u_{h} \cdot n_{F}-\beta_{h} \cdot n_{F} u_{h}\right\|_{0, F}, \\
\eta_{F}\left(u_{h}\right)=h_{F}^{\frac{1}{2}} \max \left(\alpha_{F}, \varepsilon^{\frac{1}{2}}\right)\left\|\llbracket \nabla_{h} u_{h} \rrbracket_{F}\right\|_{0, F},
\end{gathered}
$$

where $\mathscr{F}_{T}^{(1)}=\mathscr{F}_{T} \cap\left\{\mathscr{F}_{h}^{i} \cup \mathscr{F}_{h}^{\text {out }}\right\}, \mathscr{F}_{T}^{(2)}=\mathscr{F}_{T} \cap \mathscr{F}_{h}^{\text {in }}$ and $\llbracket \nabla_{h} u_{h} \rrbracket_{F} \cdot n_{F}=\partial_{h} u_{h}$ if $F \in \mathscr{F}_{h}^{\text {out }}$.

REMARK 4.1 Since $\varepsilon^{\frac{3}{4}} \alpha_{F}^{\frac{1}{2}} \leqslant h_{F}^{\frac{1}{2}} \max \left(\alpha_{F}, \varepsilon^{\frac{1}{2}}\right)$, the sum over $F \in \mathscr{F}_{T}^{(1)}$ in (4.6) can be restricted to $F \in \mathscr{F}_{T} \cap \mathscr{F}_{h}^{\text {out }}$ and the contribution of internal faces can be absorbed in $\eta_{F}\left(u_{h}\right)$. Henceforth, this redundancy is not avoided to express the a posteriori error estimate as the sum of a term which is formally identical to that obtained in a conforming setting, namely $\eta_{T}\left(u_{h}\right)$, and a term specific to nonconformity, namely $\eta_{F}\left(u_{h}\right)$.

Proof. Let $v_{h}=\mathscr{I}_{\mathrm{Os}} u_{h} \in P_{\mathrm{c}}^{1}\left(\mathscr{T}_{h}\right)$ and set $w=u-v_{h} \in H^{1}(\Omega)$. Then,

$$
\left\|u-v_{h}\right\|_{\varepsilon \beta \sigma, \Omega}^{2} \lesssim a_{h}\left(u-u_{h}, w\right)+a_{h}\left(u_{h}-v_{h}, w\right) .
$$

Furthermore, for all $w_{h} \in P_{\mathrm{c}}^{1}\left(\mathscr{T}_{h}\right)$ the following equality holds:

$$
a_{h}\left(u-u_{h}, w\right)=a_{h}\left(u-u_{h}, w-w_{h}\right)+j_{h}\left(u_{h}, w_{h}\right)+s_{h}\left(u_{h}, w_{h}\right) .
$$

Hence,

$$
\left\|u-v_{h}\right\|_{\varepsilon \beta \sigma, \Omega}^{2} \lesssim a_{h}\left(u-u_{h}, w-w_{h}\right)+j_{h}\left(u_{h}, w_{h}\right)+s_{h}\left(u_{h}, w_{h}\right)+a_{h}\left(u_{h}-v_{h}, w\right) .
$$

Let us estimate the four terms in the right-hand side of the above equation. Set $w_{h}=\mathscr{C}_{h} w \in P_{\mathrm{c}}^{1}\left(\mathscr{T}_{h}\right)$ where $\mathscr{C}_{h}$ denotes the Clément interpolant of $w$.

(1) Estimate of $a_{h}\left(u-u_{h}, w-w_{h}\right)$. Using the techniques presented in Verfürth (1998) yields

$$
a_{h}\left(u-u_{h}, w-w_{h}\right) \lesssim\left(\sum_{T \in \mathscr{T}_{h}}\left[\eta_{T}\left(u_{h}\right)^{2}+\delta_{T}\left(u_{h}\right)^{2}\right]\right)^{\frac{1}{2}}\|w\|_{\varepsilon \beta \sigma, \Omega} .
$$

(2) Estimate of $j_{h}\left(u_{h}, w_{h}\right)$. Let $F \in \mathscr{F}_{h}^{i}$.

(2.a) Assume $\alpha_{F}=1$. Owing to (2.6) and (2.7),

$$
\int_{F} \beta \cdot n_{F} \llbracket u_{h} \rrbracket_{F} w_{h}^{\downarrow} \lesssim h_{F}^{\frac{1}{2}}\left\|\llbracket \nabla_{h} u_{h} \rrbracket_{F}\right\|_{0, F}\left\|w_{h}\right\|_{0, \mathscr{T}_{F}}=h_{F}^{\frac{1}{2}} \alpha_{F}\left\|\llbracket \nabla_{h} u_{h} \rrbracket_{F}\right\|_{0, F}\left\|w_{h}\right\|_{0, \mathscr{T}_{F}} .
$$


(2.b) Assume $\alpha_{F}=\varepsilon^{-\frac{1}{2}} h_{F}$. Since $\int_{F} \llbracket u_{h} \rrbracket_{F}=0$, it follows that

$$
\int_{F} \beta \cdot n_{F} \llbracket u_{h} \rrbracket_{F} w_{h}^{\downarrow}=\int_{F}\left(\beta-\Pi_{F}^{0} \beta\right) \cdot n_{F} \llbracket u_{h} \rrbracket_{F} w_{h}^{\downarrow}+\int_{F} \Pi_{F}^{0} \beta \cdot n_{F} \llbracket u_{h} \rrbracket_{F}\left(w_{h}^{\downarrow}-\Pi_{F}^{0} w_{h}^{\downarrow}\right),
$$

where $\Pi_{F}^{0}$ is defined in the proof of Lemma 3.4. Since $\beta \in\left[\mathscr{C}^{0, \frac{1}{2}}(\Omega)\right]^{d}$, using (2.6), (2.7) and (4.2) yields

$$
\begin{aligned}
\int_{F}\left(\beta-\Pi_{F}^{0} \beta\right) \cdot n_{F} \llbracket u_{h} \rrbracket_{F} w_{h}^{\downarrow} & \lesssim h_{F}^{\frac{1}{2}}\left\|\llbracket u_{h} \rrbracket_{F}\right\|_{0, F}\left\|w_{h}^{\downarrow}\right\|_{0, F} \\
& \lesssim h_{F}\left\|\llbracket \nabla_{h} u_{h} \rrbracket_{F}\right\|_{0, F}\left\|w_{h}\right\|_{0, \mathscr{T}_{F}} \\
& \lesssim h_{F}^{\frac{1}{2}} \max \left(\varepsilon^{\frac{1}{2}}, \alpha_{F}\right)\left\|\llbracket \nabla_{h} u_{h} \rrbracket_{F}\right\|_{0, F}\left\|w_{h}\right\|_{0, \mathscr{T}_{F}} .
\end{aligned}
$$

Moreover,

$$
\begin{aligned}
\int_{F} \Pi_{F}^{0} \beta \cdot n_{F} \llbracket u_{h} \rrbracket_{F}\left(w_{h}^{\downarrow}-\Pi_{F}^{0} w_{h}^{\downarrow}\right) & \lesssim\left\|\llbracket u_{h} \rrbracket_{F}\right\|_{0, F}\left\|w_{h}^{\downarrow}-\Pi_{F}^{0} w_{h}^{\downarrow}\right\|_{0, F} \\
& \lesssim \varepsilon^{-\frac{1}{2}} h_{F}^{\frac{3}{2}}\left\|\llbracket \nabla_{h} u_{h} \rrbracket_{F}\right\|_{0, F}\left\|\varepsilon^{\frac{1}{2}} \nabla w_{h}\right\|_{0, \mathscr{T}_{F}} .
\end{aligned}
$$

Collecting the above estimates yields

$$
j_{h}\left(u_{h}, w_{h}\right) \lesssim\left(\sum_{F \in \mathscr{F}_{h}^{i}} \eta_{F}\left(u_{h}\right)^{2}\right)^{\frac{1}{2}}\left(\left\|\varepsilon^{\frac{1}{2}} \nabla w_{h}\right\|_{0, \Omega}+\left\|w_{h}\right\|_{0, \Omega}\right) .
$$

Finally, owing to the $L^{2}$ - and $H^{1}$-stability of the Clément interpolation operator (Bernardi \& Girault, 1998), it is inferred that

$$
j_{h}\left(u_{h}, w_{h}\right) \lesssim\left(\sum_{F \in \mathscr{F}_{h}^{i}} \eta_{F}\left(u_{h}\right)^{2}\right)^{\frac{1}{2}}\|w\|_{\varepsilon \beta \sigma, \Omega} .
$$

(3) Estimate of $s_{h}\left(u_{h}, w_{h}\right)$. Let $F \in \mathscr{F}_{h}^{i}$.

(3.a) Assume $\alpha_{F}=1$. Owing to (2.5) and (2.6),

$$
\int_{F} \frac{h_{F}^{2}}{\beta_{\infty, F}} \llbracket \beta \cdot \nabla_{h} u_{h} \rrbracket_{F} \llbracket \beta \cdot \nabla w_{h} \rrbracket_{F} \lesssim h_{F}^{\frac{1}{2}}\left\|\llbracket \nabla_{h} u_{h} \rrbracket_{F}\right\|_{0, F}\left\|w_{h}\right\|_{0, \mathscr{T}_{F}} .
$$

(3.b) Assume $\alpha_{F}=\varepsilon^{-\frac{1}{2}} h_{F}$. Then,

$$
\begin{aligned}
\int_{F} \frac{h_{F}^{2}}{\beta_{\infty, F}} \llbracket \beta \cdot \nabla_{h} u_{h} \rrbracket_{F} \llbracket \beta \cdot \nabla w_{h} \rrbracket_{F} & \lesssim h_{F}^{2}\left\|\llbracket \nabla_{h} u_{h} \rrbracket_{F}\right\|_{0, F} h_{F}^{-\frac{1}{2}}\left\|\nabla w_{h}\right\|_{0, \mathscr{T}_{F}} \\
& \lesssim \varepsilon^{-\frac{1}{2}} h_{F}^{\frac{3}{2}}\left\|\llbracket \nabla_{h} u_{h} \rrbracket_{F}\right\|_{0, F}\left\|\varepsilon^{\frac{1}{2}} \nabla w_{h}\right\|_{0, \mathscr{T}_{F}} .
\end{aligned}
$$


Collecting the above estimates and using the $L^{2}$ - and $H^{1}$-stability of the Clément interpolation operator yields

$$
s_{h}\left(u_{h}, w_{h}\right) \lesssim\left(\sum_{F \in \mathscr{F}_{h}^{i}} \eta_{F}\left(u_{h}\right)^{2}\right)^{\frac{1}{2}}\|w\|_{\varepsilon \beta \sigma, \Omega}
$$

(4) Estimate of $a_{h}\left(u_{h}-v_{h}, w\right)$.

(4.a) Estimate of the diffusive term. Let $T \in \mathscr{T}_{h}$. Use (2.10) to infer

$$
\int_{T} \varepsilon \nabla_{h}\left(u_{h}-v_{h}\right) \cdot \nabla w \lesssim\left(\sum_{F \in \mathscr{F}_{T}^{\mathrm{OS}}} \varepsilon^{\frac{1}{2}} h_{F}^{\frac{1}{2}}\left\|\llbracket \nabla_{h} u_{h} \rrbracket_{F}\right\|_{0, F}\right)\left\|\varepsilon^{\frac{1}{2}} \nabla w\right\|_{0, T} .
$$

(4.b) Estimate of the reactive term. Let $T \in \mathscr{T}_{h}$. Use (2.10) and (4.3) to infer

$$
\begin{aligned}
\int_{T} \theta\left(u_{h}-v_{h}\right) w & \lesssim\left(\sum_{F \in \mathscr{F}_{T}^{\mathrm{OS}}} h_{F}^{\frac{3}{2}}\left\|\llbracket \nabla_{h} u_{h} \rrbracket_{F}\right\|_{0, F}\right)\|w\|_{0, T} \\
& \lesssim\left(\sum_{F \in \mathscr{F}_{T}^{\mathrm{OS}}} h_{F}^{\frac{1}{2}} \alpha_{F}\left\|\llbracket \nabla_{h} u_{h} \rrbracket_{F}\right\|_{0, F}\right)\|w\|_{0, T}
\end{aligned}
$$

(4.c) Estimate of the advective and face terms. Observe that these terms can be written in the form $\sum_{T \in \mathscr{T}_{h}} \Xi_{T}$ with

$$
\begin{aligned}
\Xi_{T} & =\int_{T} \beta \cdot \nabla_{h}\left(u_{h}-v_{h}\right) w-\int_{\partial T \cap \partial \Omega_{\mathrm{in}}}\left(\beta \cdot n_{T}\right)\left(u_{h}-v_{h}\right) w \\
& =-\int_{T}\left(u_{h}-v_{h}\right) \beta \cdot \nabla w-\int_{T}(\nabla \cdot \beta)\left(u_{h}-v_{h}\right) w+\int_{\partial T^{*}}\left(\beta \cdot n_{T}\right)\left(u_{h}-v_{h}\right) w,
\end{aligned}
$$

where $n_{T}$ denotes the outward normal to $T$ and $\partial T^{*}=\partial T \backslash\left(\partial T \cap \partial \Omega_{\text {in }}\right)$. If $\alpha_{T}=1$, consider (4.8) and use (2.10) to infer

$$
\begin{aligned}
\int_{T} \beta \cdot \nabla_{h}\left(u_{h}-v_{h}\right) w & \lesssim\left(\sum_{F \in \mathscr{F}_{T}^{\mathrm{Os}}} h_{F}^{\frac{1}{2}}\left\|\llbracket \nabla_{h} u_{h} \rrbracket_{F}\right\|_{0, F}\right)\|w\|_{0, T} \\
& \lesssim\left(\sum_{F \in \mathscr{F}_{T}^{\mathrm{Os}}} h_{F}^{\frac{1}{2}} \alpha_{F}\left\|\llbracket \nabla_{h} u_{h}\right\|_{F} \|_{0, F}\right)\|w\|_{0, T}
\end{aligned}
$$


owing to the shape-regularity of the mesh family. Moreover, if $T$ has a face on $\partial \Omega_{\text {in }}$, say $F_{T}$, using (2.6) and (2.10) leads to

$$
\int_{\partial T \cap \partial \Omega_{\mathrm{in}}}\left(\beta \cdot n_{T}\right)\left(u_{h}-v_{h}\right) w \lesssim\left(\sum_{F \in \mathscr{F}_{T}^{\mathrm{Os}}} h_{F}\left\|\llbracket \nabla_{h} u_{h} \rrbracket_{F}\right\|_{0, F}\right)\left\||\beta \cdot n|^{\frac{1}{2}} w\right\|_{0, F_{T}} .
$$

Hence,

$$
\left|\Xi_{T}\right| \lesssim\left(\sum_{F \in \mathscr{F}_{T}^{\mathrm{Os}}} h_{F}^{\frac{1}{2}} \alpha_{F}\left\|\llbracket \nabla_{h} u_{h} \rrbracket_{F}\right\|_{0, F}\right)\left(\|w\|_{0, T}+\left\||\beta \cdot n|^{\frac{1}{2}} w\right\|_{0, F_{T}}\right) .
$$

If $\alpha_{T}=\varepsilon^{-\frac{1}{2}} h_{T}$, consider (4.9). Owing to (2.10),

$$
\int_{T}\left(u_{h}-v_{h}\right) \beta \cdot \nabla w \lesssim\left(\sum_{F \in \mathscr{F}_{T}^{\mathrm{Os}}} \varepsilon^{-\frac{1}{2}} h_{F}^{\frac{3}{2}}\left\|\llbracket \nabla_{h} u_{h} \rrbracket_{F}\right\|_{0, F}\right)\left\|\varepsilon^{\frac{1}{2}} \nabla w\right\|_{0, T} .
$$

Furthermore, the term $\int_{T}(\nabla \cdot \beta)\left(u_{h}-v_{h}\right) w$ is estimated as in Step (4.b). Let $F \subset \partial T^{*}$. Assume first that $F \in \mathscr{F}_{h}^{i}$. Observe that

$$
\int_{F} \beta \cdot n_{F} \llbracket u_{h}-v_{h} \rrbracket_{F} w=\int_{F}\left(\beta-\Pi_{F}^{0} \beta\right) \cdot n_{F} \llbracket u_{h} \rrbracket_{F} w+\int_{F} \Pi_{F}^{0} \beta \cdot n_{F} \llbracket u_{h} \rrbracket_{F}\left(w-\Pi_{F}^{0} w\right),
$$

since $v_{h} \in P_{\mathrm{c}}^{1}\left(\mathscr{T}_{h}\right)$. Proceeding as above yields

$$
\begin{aligned}
\int_{F} \beta \cdot n_{F} \llbracket u_{h}-v_{h} \rrbracket_{F} w & \lesssim h_{F}^{\frac{3}{2}}\left\|\llbracket \nabla_{h} u_{h} \rrbracket_{F}\right\|_{0, F}\|w\|_{0, F}+\varepsilon^{-\frac{1}{2}} h_{F}^{\frac{3}{2}}\left\|\llbracket \nabla_{h} u_{h} \rrbracket_{F}\right\|_{0, F}\left\|\varepsilon^{\frac{1}{2}} \nabla w\right\|_{0, \mathscr{T}_{F}} \\
& \lesssim \varepsilon^{-\frac{1}{2}} h_{F}^{\frac{3}{2}}\left\|\llbracket \nabla_{h} u_{h} \rrbracket_{F}\right\|_{0, F}\left(\left\|\varepsilon^{\frac{1}{2}} \nabla w\right\|_{0, \mathscr{T}_{F}}+\|w\|_{0, \mathscr{T}_{F}}\right),
\end{aligned}
$$

where we have used the trace inequality $\|w\|_{0, F} \lesssim\|w\|_{0, \mathscr{T}_{F}}^{\frac{1}{2}}\|w\|_{1, \mathscr{T}_{F}}^{\frac{1}{2}}$ valid for all $w \in$ $H^{1}(\Omega)$. Furthermore, if $F \subset \partial \Omega_{\text {out }}$, using (2.6), (2.10) and (4.2) yields

$$
\begin{aligned}
\int_{F} \beta \cdot n\left(u_{h}-v_{h}\right) w & \lesssim\left\|u_{h}-v_{h}\right\|_{0, F}\left\||\beta \cdot n|^{\frac{1}{2}} w\right\|_{0, F} \\
& \lesssim\left(\sum_{F^{\prime} \in \mathscr{F}_{T(F)}^{\mathrm{Os}}} h_{F^{\prime}}\left\|\llbracket \nabla_{h} u_{h} \rrbracket_{F^{\prime}}\right\|_{0, F^{\prime}}\right)\left\||\beta \cdot n|^{\frac{1}{2}} w\right\|_{0, F} \\
& \lesssim\left(\sum_{F^{\prime} \in \mathscr{F}_{T(F)}^{\mathrm{Os}}} h_{F^{\prime}}^{\frac{1}{2}} \max \left(\varepsilon^{\frac{1}{2}}, \alpha_{F^{\prime}}\right)\left\|\llbracket \nabla_{h} u_{h} \rrbracket_{F^{\prime}}\right\|_{0, F^{\prime}}\right)\left\||\beta \cdot n|^{\frac{1}{2}} w\right\|_{0, F}
\end{aligned}
$$


Collecting the above inequalities yields

$$
a_{h}\left(u_{h}-v_{h}, w\right) \lesssim\left(\sum_{F \in \mathscr{F}_{h}^{i}} \eta_{F}\left(u_{h}\right)^{2}\right)^{\frac{1}{2}}\|w\|_{\varepsilon \beta \sigma, \Omega}
$$

(5) Owing to Steps (1)-(4) above, it is inferred that

$$
\left\|u-v_{h}\right\|_{\varepsilon \beta \sigma, \Omega} \lesssim\left(\sum_{T \in \mathscr{T}_{h}}\left[\eta_{T}\left(u_{h}\right)^{2}+\delta_{T}\left(u_{h}\right)^{2}\right]+\sum_{F \in \mathscr{F}_{h}^{i}} \eta_{F}\left(u_{h}\right)^{2}\right)^{\frac{1}{2}} .
$$

Using (2.10), (4.3) and the shape-regularity of the mesh family yields

$$
\begin{aligned}
\left\|\varepsilon^{\frac{1}{2}} \nabla_{h}\left(u_{h}-v_{h}\right)\right\|_{0, \Omega}+\left\|u_{h}-v_{h}\right\|_{0, \Omega} & \lesssim\left(\sum_{F \in \mathscr{F}_{h}^{i}}\left(\varepsilon h_{F}+h_{F}^{3}\right)\left\|\llbracket \nabla_{h} u_{h} \rrbracket_{F}\right\|_{0, F}^{2}\right)^{\frac{1}{2}} \\
& \lesssim\left(\sum_{F \in \mathscr{F}_{h}^{i}}\left(\varepsilon h_{F}+h_{F} \alpha_{F}^{2}\right)\left\|\llbracket \nabla_{h} u_{h} \rrbracket_{F}\right\|_{0, F}^{2}\right)^{\frac{1}{2}} .
\end{aligned}
$$

Moreover, for all $F \in \mathscr{F}_{h}^{\partial}$, owing to (2.6), (2.10) and (4.2),

$$
\begin{aligned}
\left\||\beta \cdot n|^{\frac{1}{2}}\left(u_{h}-v_{h}\right)\right\|_{0, F} & \lesssim \sum_{F^{\prime} \in \mathscr{F}_{T(F)}^{\mathrm{SS}}} h_{F}\left\|\llbracket \nabla_{h} u_{h} \rrbracket_{F^{\prime}}\right\|_{0, F^{\prime}} \\
& \lesssim \sum_{F^{\prime} \in \mathscr{F}_{T(F)}^{\mathrm{OS}}} h_{F^{\prime}}^{\frac{1}{2}} \max \left(\varepsilon^{\frac{1}{2}}, \alpha_{F^{\prime}}\right)\left\|\llbracket \nabla_{h} u_{h} \rrbracket_{F^{\prime}}\right\|_{0, F^{\prime}} .
\end{aligned}
$$

Collecting the above estimates yields

$$
\left\|u_{h}-v_{h}\right\|_{\varepsilon \beta \sigma, \Omega} \lesssim\left(\sum_{F \in \mathscr{F} h} \eta_{F}\left(u_{h}\right)^{2}\right)^{\frac{1}{2}}
$$

Use the triangle inequality to conclude.

Let $T \in \mathscr{T}_{h}$ and let $\Delta_{T}$ denote the union of elements of $\mathscr{T}_{h}$ sharing at least a vertex with $T$. For all $w \in V(h)$, localize $\|w\|_{\varepsilon \beta \sigma, \Omega}$ as follows:

$$
\|w\|_{\varepsilon \beta \sigma, \Delta_{T}}=\left\|\varepsilon^{\frac{1}{2}} \nabla_{h} v\right\|_{0, \Delta_{T}}+\left\|\sigma^{\frac{1}{2}} w\right\|_{0, \Delta_{T}}+\left(\sum_{F \in \mathscr{F}_{\Delta_{T}} \cap \mathscr{F}_{h}^{\partial}}\left\||\beta \cdot n|^{\frac{1}{2}} w\right\|_{0, F}^{2}\right)^{\frac{1}{2}},
$$

where $\mathscr{F}_{\Delta_{T}}$ denotes the set of faces of the elements in $\Delta_{T}$. 
THEOREM 4.2 (Lower bound) For all $T \in \mathscr{T}_{h}$,

$$
\eta_{T}\left(u_{h}\right) \lesssim\left(1+\varepsilon^{-\frac{1}{2}} \alpha_{T}\right)\left\|u-u_{h}\right\|_{\varepsilon \beta \sigma, \Delta_{T}}+\delta_{\Delta_{T}}\left(u_{h}\right),
$$

where $\delta_{\Delta_{T}}\left(u_{h}\right)=\sum_{T^{\prime} \in \Delta_{T}} \delta_{T^{\prime}}\left(u_{h}\right)$, and for all $F \in \mathscr{F}_{h}^{i}$,

$$
\eta_{F}\left(u_{h}\right) \lesssim \varepsilon^{-\frac{1}{2}} \max \left(\alpha_{F}, \varepsilon^{\frac{1}{2}}\right)\left(\left\|u-u_{h}\right\|_{\varepsilon \beta \sigma, \mathscr{T}_{F}}+\inf _{z_{h} \in\left[P_{\mathrm{c}}^{1}\left(\mathscr{T}_{h}\right)\right]^{d}}\left\|\varepsilon^{\frac{1}{2}}\left(\nabla u-z_{h}\right)\right\|_{0, \mathscr{T}_{F}}\right) .
$$

Proof. The upper bound (4.10) is obtained by using the techniques presented in Verfürth (1998). To prove (4.11), let $z_{h} \in\left[P_{\mathrm{c}}^{1}\left(\mathscr{T}_{h}\right)\right]^{d}$ and let $F \in \mathscr{F}_{h}^{i}$. Observe that $\left[\nabla_{h} u_{h} \rrbracket_{F}=\llbracket \nabla_{h} u_{h}-z_{h} \rrbracket_{F}\right.$. Then, using (2.6) and the triangle inequality yields

$$
\left\|\llbracket \nabla_{h} u_{h} \rrbracket_{F}\right\|_{0, F} \lesssim h_{F}^{-\frac{1}{2}}\left\|\nabla_{h} u_{h}-z_{h}\right\|_{0, \mathscr{T}_{F}} \lesssim h_{F}^{-\frac{1}{2}}\left(\left\|\nabla u-\nabla_{h} u_{h}\right\|_{0, \mathscr{T}_{F}}+\left\|\nabla u-z_{h}\right\|_{0, \mathscr{T}_{F}}\right) .
$$

The conclusion is straightforward.

REMARK 4.2 Since $\alpha_{F} \leqslant 1$, the following global lower bound is inferred:

$$
\varepsilon^{\frac{1}{2}}\left(\sum_{F \in \mathscr{F}_{h}^{i}} \eta_{F}\left(u_{h}\right)^{2}\right)^{\frac{1}{2}} \lesssim\left\|u-u_{h}\right\|_{\varepsilon \beta \sigma, \Omega}+\inf _{z_{h} \in\left[P_{\mathrm{c}}^{1}\left(\mathscr{T}_{h}\right)\right]^{d}}\left\|\varepsilon^{\frac{1}{2}}\left(\nabla u-z_{h}\right)\right\|_{0, \Omega} .
$$

Hence, if the exact solution is smooth enough $\left(u \in H^{2}(\Omega)\right)$, the first term in the right-hand side is bounded by $h\left(\varepsilon^{\frac{1}{2}}+h^{\frac{1}{2}}\right)\|u\|_{2, \Omega}$ owing to Theorem 3.1 , while the second term is bounded by $h \varepsilon^{\frac{1}{2}}\|u\|_{2, \Omega}$.

\section{Numerical results}

In this section, two test cases are presented to illustrate the above theoretical results. In both cases, $\Omega=(0,1) \times(0,1)$ and we consider a shape-regular family of unstructured triangulations of $\Omega$ with mesh size $h_{i}=h_{0} \times 2^{-i}$ with $h_{0}=0.1$ and $i \in\{0, \ldots, 4\}$. The diffusion coefficient $\varepsilon$ takes the values $\left\{10^{-2}, 10^{-4}, 10^{-6}\right\}$ and the reaction coefficient $\theta$ is set to 1 . The parameter $\gamma$ in (2.15) is set to 0.005 . While the optimal value for this parameter is problem-dependent, our experience with various test cases shows that the stabilized method behaves fairly well with values in the range $10^{-3}-10^{-2}$, and that within this range, the method is less sensitive to the actual choice for the value of $\gamma$ than is the streamline-diffusion method to the choice of its corresponding parameter.

\subsection{Test case 1}

Let $\beta=(1,0)^{\mathrm{T}}$ and choose the data $f$ and $g$ so that the exact solution of (2.1) is

$$
u(x, y)=\frac{1}{2}\left(1-\tanh \left(\frac{0.5-x}{a_{w}}\right)\right),
$$

with internal layer width $a_{w}=0.05$.

Table 1 presents the convergence results for the error $\left\|u-u_{h}\right\|_{A, \Omega} ; N_{\text {fa }}$ denotes the number of degrees of freedom (i.e. the number of mesh faces) and $\omega$ denotes the convergence order with respect to 
TABLE 1 Numerical errors and convergence orders for the different values of $\varepsilon$

\begin{tabular}{|c|c|c|c|c|c|c|c|}
\hline \multicolumn{2}{|c|}{ Mesh } & \multicolumn{2}{|c|}{$\varepsilon=10^{-2}$} & \multicolumn{2}{|c|}{$\varepsilon=10^{-4}$} & \multicolumn{2}{|l|}{$\varepsilon=10^{-6}$} \\
\hline$i$ & $N_{\mathrm{fa}}$ & $\left\|u-u_{h}\right\|_{A, \Omega}$ & $\omega$ & $\left\|u-u_{h}\right\|_{A, \Omega}$ & $\omega$ & $\left\|u-u_{h}\right\|_{A, \Omega}$ & $\omega$ \\
\hline 0 & 374 & 1.04 & 一 & 1.01 & - & $9.99 \times 10^{-1}$ & - \\
\hline 1 & 1441 & $4.05 \times 10^{-1}$ & 1.40 & $3.76 \times 10^{-1}$ & 1.46 & $3.71 \times 10^{-1}$ & 1.47 \\
\hline 2 & 5621 & $1.53 \times 10^{-1}$ & 1.43 & $1.29 \times 10^{-1}$ & 1.57 & $1.26 \times 10^{-1}$ & 1.59 \\
\hline 3 & 22330 & $6.02 \times 10^{-2}$ & 1.35 & $4.52 \times 10^{-2}$ & 1.52 & $4.40 \times 10^{-2}$ & 1.52 \\
\hline 4 & 88961 & $2.45 \times 10^{-2}$ & 1.30 & $1.61 \times 10^{-2}$ & 1.49 & $1.55 \times 10^{-2}$ & 1.51 \\
\hline
\end{tabular}

the square root of the number of degrees of freedom (or, equivalently, the mesh size for quasi-uniform meshes). In the advection-dominated regime $\left(\varepsilon=10^{-4}\right.$ and $\left.\varepsilon=10^{-6}\right)$, the error decreases as $h^{\frac{3}{2}}$. In the intermediate regime $\left(\varepsilon=10^{-2}\right.$ ), the convergence order changes from $\frac{3}{2}$ to 1 as the mesh is refined. These results are in agreement with the estimate derived in Theorem 3.1.

Let $\eta_{1}\left(u_{h}\right), \eta_{2}\left(u_{h}\right)$ and $\delta\left(u_{h}\right)$ be the global error estimators defined as

$$
\eta_{1}\left(u_{h}\right)=\left(\sum_{T \in \mathscr{T}_{h}} \eta_{T}\left(u_{h}\right)^{2}\right)^{\frac{1}{2}}, \quad \eta_{2}\left(u_{h}\right)=\left(\sum_{F \in \mathscr{F}_{h}^{i}} \eta_{F}\left(u_{h}\right)^{2}\right)^{\frac{1}{2}}, \quad \delta\left(u_{h}\right)=\left(\sum_{T \in \mathscr{T}_{h}} \delta_{T}\left(u_{h}\right)^{2}\right)^{\frac{1}{2}},
$$

where the local error indicators $\eta_{T}\left(u_{h}\right), \eta_{F}\left(u_{h}\right)$ and $\delta_{T}\left(u_{h}\right)$ are defined in (4.6), (4.7) and (4.5), respectively. Since the data are smooth, the data error indicator $\delta\left(u_{h}\right)$ is evaluated using $L^{2}$-orthogonal projections onto the space of (discontinuous) piecewise affine functions. The asymptotic behaviour of the global error estimators with respect to the number of degrees of freedom is presented in Fig. 1. The error $u-u_{h}$ measured in the norm $\|\cdot\|_{\varepsilon \beta \sigma, \Omega}$ is also presented in Fig. 1. For the three values of the diffusion coefficient, the error estimator $\eta_{1}\left(u_{h}\right)$ has approximately the same convergence order as the error. The data error indicator $\delta\left(u_{h}\right)$ converges to second order in the diffusion-dominated regime and to order $\frac{3}{2}$ in the advection-dominated regime. The error estimator $\eta_{2}\left(u_{h}\right)$ super-converges in the diffusiondominated regime, while its convergence order is close to 1 in the advection-dominated regime. The efficiency index evaluated as

$$
I=\frac{\eta_{1}\left(u_{h}\right)+\eta_{2}\left(u_{h}\right)+\delta\left(u_{h}\right)}{\left\|u-u_{h}\right\|_{\varepsilon \beta \sigma, \Omega}}
$$

is in the range 8-30 for $\varepsilon=10^{-2}$, in the range 73-280 for $\varepsilon=10^{-4}$ and in the range 97-1262 for $\varepsilon=10^{-6}$. The increase of the efficiency index is roughly proportional to $\varepsilon^{-\frac{1}{2}}$, in agreement with the theoretical results of Section 4.

\subsection{Test case 2}

Let $\Gamma_{1}$ denote the lower horizontal edge of $\Omega$ and let $\Gamma_{2}$ denote its left vertical edge. Set $\beta=(2,1)^{\mathrm{T}}$, $f=0$, and define the boundary data $g$ on $\Gamma_{1} \cup \Gamma_{2}$ such that

$$
g(x, y)=\frac{1}{2}\left(\tanh \left(\frac{x}{a_{w}}\right)+1\right)+\frac{1}{2}\left(\tanh \left(-\frac{y}{a_{w}}\right)+1\right),
$$



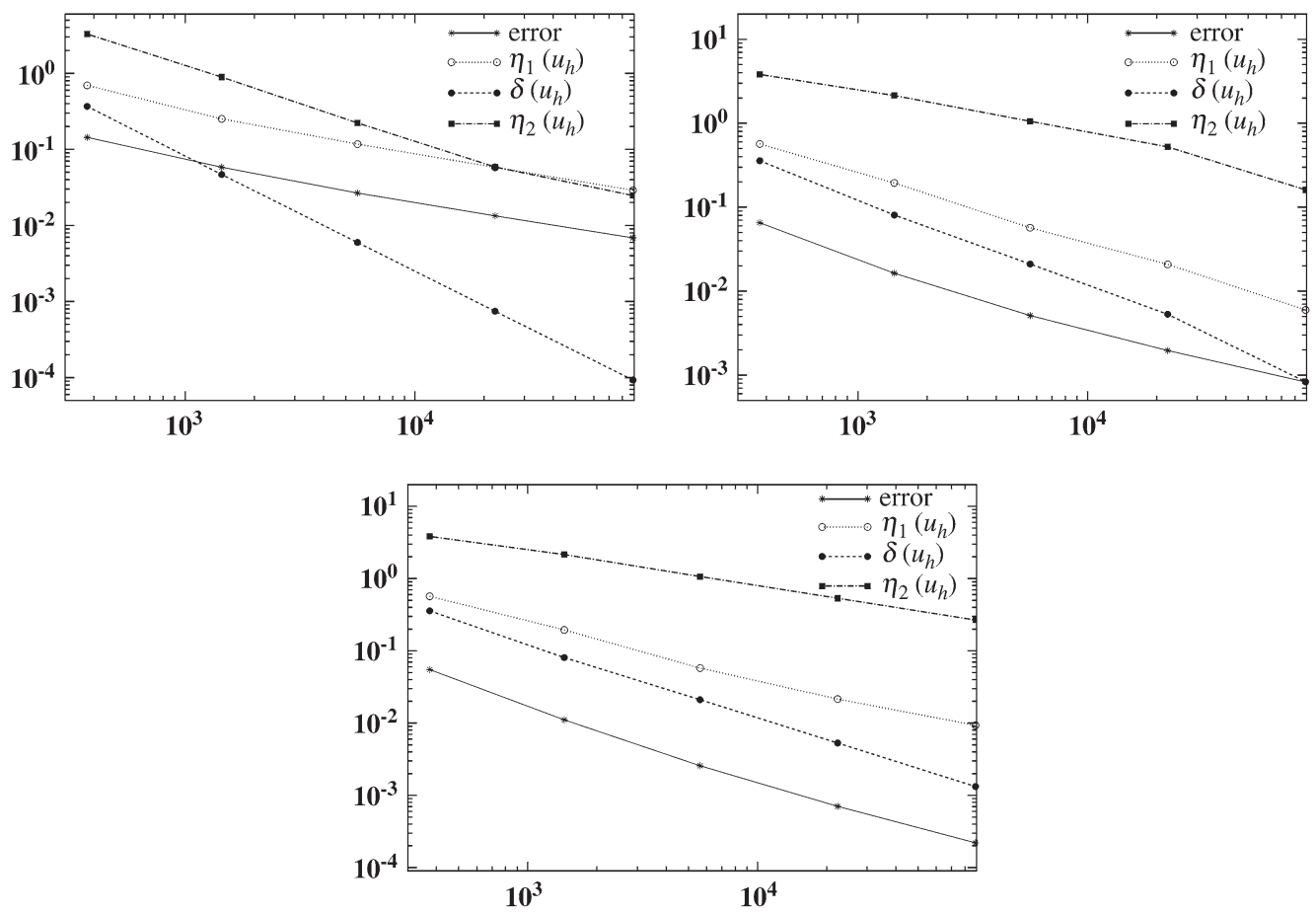

FIG. 1. Exact error and global error estimators against degrees of freedom. Top left: $\varepsilon=10^{-2}$; top right: $\varepsilon=10^{-4}$; bottom: $\varepsilon=10^{-6}$.
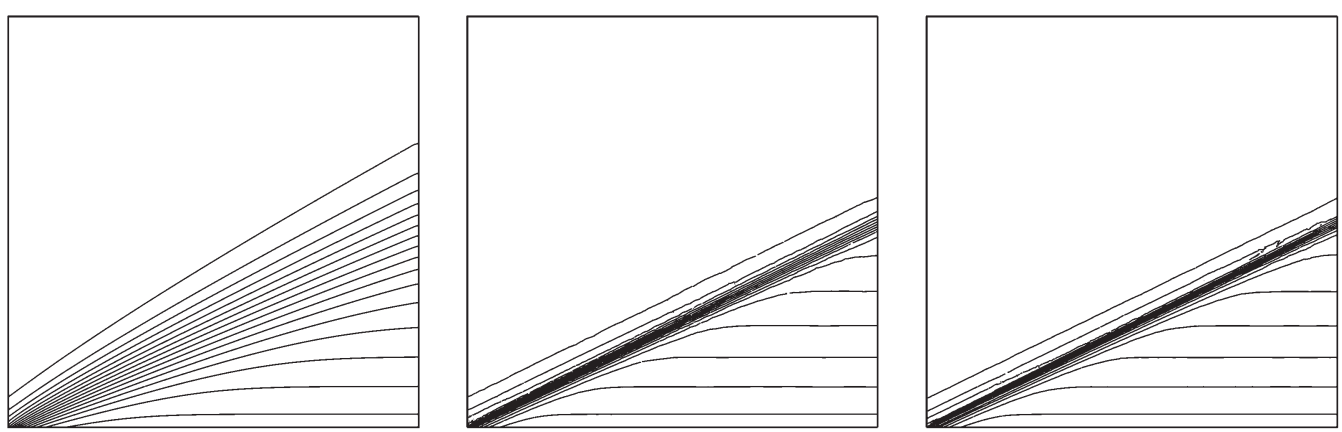

FIG. 2. Contour lines of the solution for test case 2. Left: $\varepsilon=10^{-2}$; centre: $\varepsilon=10^{-4}$; right: $\varepsilon=10^{-6}$.

with $a_{w}=0.05$. Thus, $g$ ensures a sharp but smooth transition from 0 (on $\Gamma_{2}$ ) to 1 (on $\Gamma_{1}$ ) at the origin. Owing to the Robin boundary condition, the solution exhibits an inner layer located along the line $\{x=2 y\}$. Figure 2 presents the contour lines of the computed solution for the different values of $\varepsilon$. Owing to the maximum principle, the exact solution is comprised between -1 and 0 . On the finest mesh, the approximate solution exhibits an overshoot (respectively, undershoot) of $2 \times 10^{-5}$ (respectively, 0 ) for $\varepsilon=10^{-2}, 8 \times 10^{-2}$ (respectively, $1 \times 10^{-1}$ ) for $\varepsilon=10^{-4}$ and $1 \times 10^{-1}$ (respectively, $1 \times 10^{-1}$ ) for $\varepsilon=10^{-6}$. 
To refine the mesh adaptively using the local error indicator $\eta_{T}\left(u_{h}\right)$, the following algorithm is considered:

(i) Construct an initial mesh $\mathscr{T}_{h}^{0}$. Set $i:=0$.

(ii) Compute the approximate solution $u_{h}^{i}$ on $\mathscr{T}_{h}^{i}$ and compute the local error indicators $\eta_{T_{i}}\left(u_{h}^{i}\right)$ for all $T_{i} \in \mathscr{T}_{h}^{i}$.

(iii) If the global error is sufficiently small, stop; otherwise, compute the quantities

$$
\hat{h}_{T_{i}}=l\left(\eta_{T_{i}}\left(u_{h}^{i}\right)\right) h_{T_{i}},
$$

where $l\left(\eta_{T_{i}}\left(u_{h}^{i}\right)\right)=\frac{1}{2}$ if $\eta_{T_{i}}\left(u_{h}^{i}\right) \leqslant S_{i}$ and $l\left(\eta_{T_{i}}\left(u_{h}^{i}\right)\right)=1$ otherwise. The threshold $S_{i}$ is evaluated as $S_{i}=\frac{1}{2 n t_{i}} \sum_{T_{i} \in \mathscr{T}_{h}^{i}} \eta_{T_{i}}\left(u_{h}^{i}\right)$ where $n t_{i}$ denotes the number of triangles in the mesh $\mathscr{T}_{h}^{i}$.

(iv) Using the quantities $\hat{h}_{T_{i}}$ to construct a new mesh $\mathscr{T}_{h}^{i+1}$. Go to Step (ii).

Figure 3 presents the adaptively refined meshes after five iterations of the above algorithm. For the three values of the diffusion coefficient, the mesh is refined at the origin. In the diffusion-dominated regime, the mesh is refined around the inner layer and at the outflow layer. In the advection-dominated regime, the meshes are refined along the inner layer. The refined zone becomes smaller as the diffusion coefficient $\varepsilon$ takes smaller values, indicating that the local error indicator $\eta_{T}\left(u_{h}\right)$ alone can detect the inner layer.

Figure 4 presents the asymptotic behaviour of the global error estimators $\eta_{1}\left(u_{h}\right)$ and $\eta_{2}\left(u_{h}\right)$ as a function of the number of degrees of freedom in the adaptively refined meshes. For of comparison the error estimators evaluated by setting $\alpha_{S}=\varepsilon^{-\frac{1}{2}} h_{S}$ and designated by a star superscript are also plotted. In the diffusion-dominated regime $\left(\varepsilon=10^{-2}\right), \eta_{1}\left(u_{h}\right)=\eta_{1}^{*}\left(u_{h}\right)$ and $\eta_{2}\left(u_{h}\right)=\eta_{2}^{*}\left(u_{h}\right)$ (hence, only two curves are visible in the top left plot in Fig. 4). Moreover, both error estimators super-converge on the coarser meshes, and their convergence order relaxes to the expected asymptotic behaviour (order 1) on the finer meshes. The change from advection-dominated to diffusion-dominated regime is clearly visible for $\varepsilon=10^{-4}$; the convergence order of both error estimators is close to 2 (yielding super-convergence) whenever $\alpha_{S}$ is evaluated as $\varepsilon^{-\frac{1}{2}} h_{S}$. The same behaviour is observed for $\varepsilon=10^{-6}$.
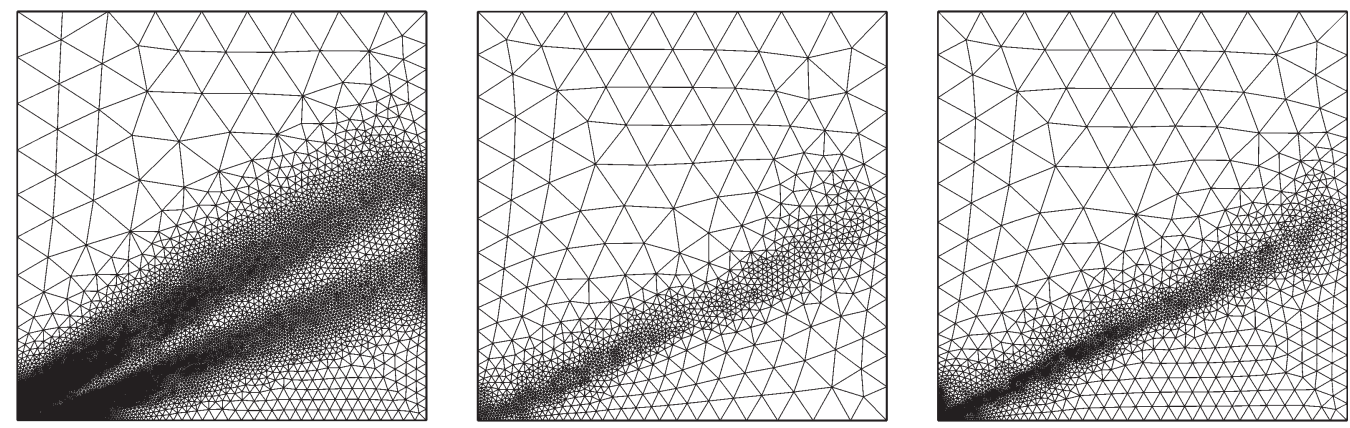

FIG. 3. Adaptive meshes after five iterations. Left: $\varepsilon=10^{-2}$ and $N_{\mathrm{fa}}=26698$; centre: $\varepsilon=10^{-4}$ and $N_{\mathrm{fa}}=7460$; right: $\varepsilon=10^{-6}$ and $N_{\text {fa }}=7158$. 

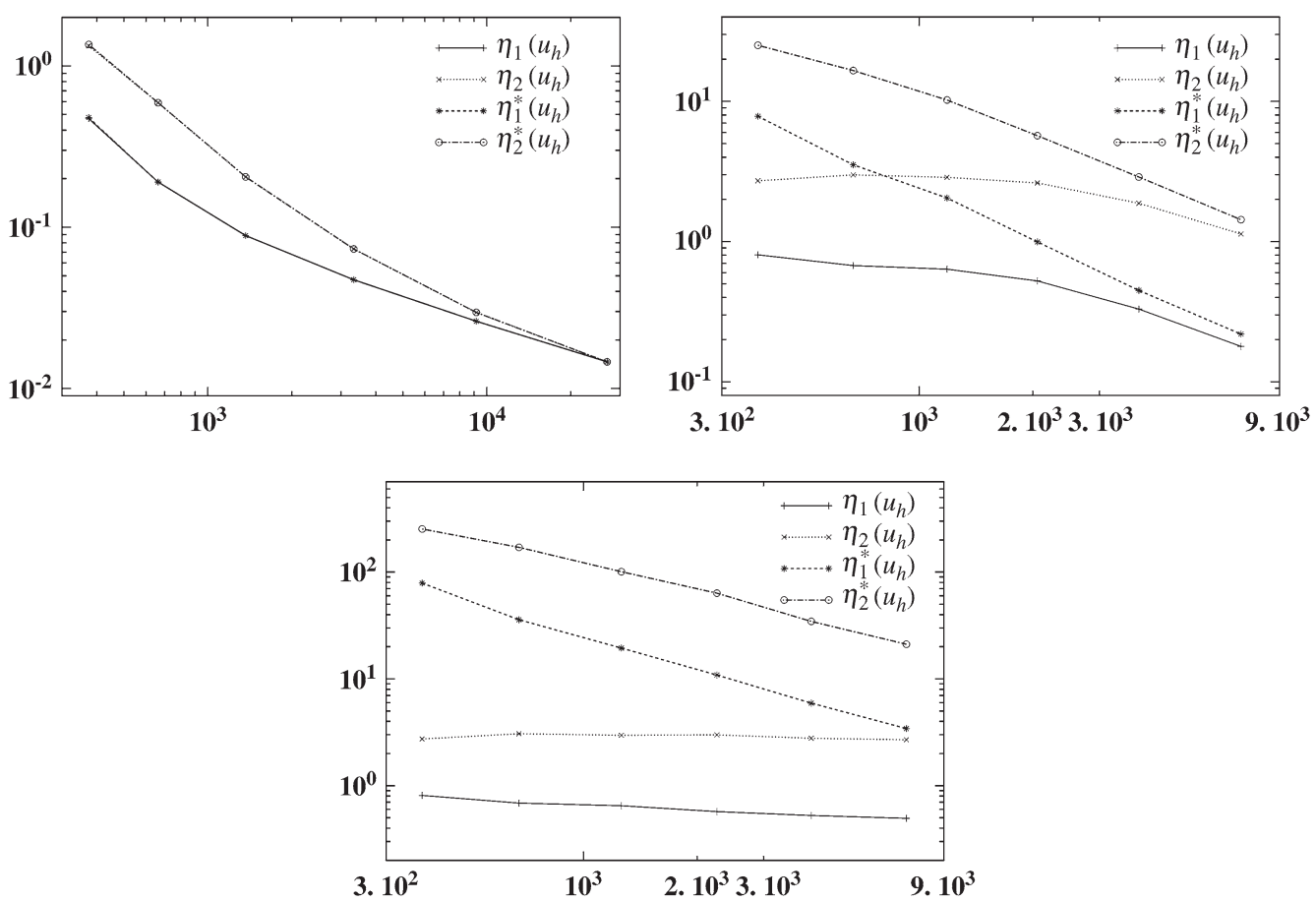

FIG. 4. Global error estimators against degrees of freedom. Top left: $\varepsilon=10^{-2}$; top right: $\varepsilon=10^{-4}$; bottom: $\varepsilon=10^{-6}$.

\section{Conclusions}

In this paper, we have presented an a priori and an a posteriori error analysis for a non-conforming finiteelement method to approximate advection-diffusion equations. The method is stabilized by penalizing the jumps of the solution and those of its advective derivative across mesh interfaces. The a priori error analysis leads to (quasi-)optimal error estimates in the mesh size in the sense that keeping the Péclet number fixed the estimates are sub-optimal of order $\frac{1}{2}$ in the $L^{2}$-norm and optimal in the broken graph norm for quasi-uniform meshes. These estimates are similar to those obtained with other methods. A drawback of the present scheme is the presence of face-oriented bilinear forms leading to a discretization stencil larger than that resulting from the use of the Crouzeix-Raviart finite element. When solving non-linear problems, e.g. the Navier-Stokes equations, these terms can be treated in the framework of a non-linear iterative solver thus avoiding the widening of the stencil; see, e.g. Ouazzi \& Turek (2005). Finally, the a posteriori error analysis leads to semi-robust error indicators, meaning that the factor between the lower and upper bounds scales as the square root of the Péclet number. The present analysis provides the first semi-robust a posteriori error estimator in a non-conforming setting and can be viewed as a first step towards establishing robust a posteriori error estimators in this setting.

\section{Acknowledgements}

This work was partly supported by the GdR MoMaS (CNRS-2439, ANDRA, BRGM, CEA, EdF). The authors are thankful to the referees for valuable suggestions. 


\section{REFERENCES}

Achdou, Y., Bernardi, C. \& Coquel, F. (2003) A priori and a posteriori analysis of finite volume discretizations of Darcy's equations. Numer. Math., 96, 17-42.

Araya, R., Behrens, E. \& Rodriguez, R. (2005a) An adaptive stabilized finite element scheme for the advection-diffusion equation. Appl. Numer. Math., 54, 491-503.

Araya, R., PozA, A. \& Stephan, E. P. (2005b) A hierarchical a posteriori error estimate for an advectiondiffusion-reaction problem. Math. Models Methods Appl. Sci., 15, 1119-1139.

Bernardi, C. \& Girault, V. (1998) A local regularization operator for triangular and quadrilateral finite elements. SIAM J. Numer. Anal., 35, 1893-1916.

Berrone, S. (2002) Robustness in a posteriori error analysis for FEM flow models. Numer. Math., 91, 389-422.

Boman, M. (2000) A posteriori error analysis in the maximum norm for a penalty finite element method for the time dependent obstacle problem. Technical Report 2000-12. Göteborg, Sweden: Chalmers Finite Element Center, Chalmers University of Technology.

BrezzI, F. \& Russo, A. (1994) Choosing bubbles for advection-diffusion problems. Math. Models Methods Appl. Sci., 4, 571-587.

Brooks, A. \& Hughes, T. (1982) Streamline upwind/Petrov-Galerkin formulations for convective dominated flows with particular emphasis on the incompressible Navier-Stokes equations. Comput. Methods Appl. Mech. Eng., 32, 199-259.

BURMAN, E. (2005) A unified analysis for conforming and non-conforming stabilized finite element methods using interior penalty. SIAM J. Numer. Anal., 43, 2012-2033.

BURMAN, E. \& HANSBO, P. (2004) Edge stabilization for Galerkin approximations of convection-diffusionreaction problems. Comput. Methods Appl. Mech. Eng., 193, 1437-1453.

Burman, E. \& HAnsbo, P. (2006) Edge stabilization for Galerkin approximations of the generalized Stokes' problem. Comput. Methods Appl. Mech. Eng., 195, 2393-2410.

Courbet, B. \& Croisille, J.-P. (1998) Finite volume box schemes on triangular meshes. ESAIM Math. Model. Numer. Anal., 32, 631-649.

Croisille, J.-P. (2000) Finite volume box schemes and mixed methods. ESAIM Math. Model. Numer. Anal., 31, $1087-1106$.

Crouzeix, M. \& Raviart, P.-A. (1973) Conforming and nonconforming mixed finite element methods for solving the stationary Stokes equations I. RAIRO Modél. Math. Anal. Numér., 3, 33-75.

Douglas, JR., J. \& Dupont, T. (1976) Interior penalty procedures for elliptic and parabolic Galerkin methods. Computing Methods in Applieds Sciences (R. Glowinski \& J. L. Lions eds). Berlin: Springer, pp. 207-216.

El ALAOUi, L. \& ERn, A. (2004) Residual and hierarchical a posteriori error estimates for nonconforming mixed finite element methods. ESAIM Math. Model. Numer. Anal., 38, 903-929.

El Alaoui, L. \& ERN, A. (2006) Nonconforming finite element methods with subgrid viscosity applied to advection-diffusion-reaction equations. Numer. Methods Partial Differ. Equ. (published online).

GUERMOND, J.-L. (1999) Stabilization of Galerkin approximations of transport equations by subgrid modeling. ESAIM Math. Model. Numer. Anal., 33, 1293-1316.

GUERMOND, J.-L. (2001) Subgrid stabilization of Galerkin approximations of linear monotone operators. IMA J. Numer. Anal., 21, 165-197.

Hoppe, R. H. W. \& Wohlmuth, B. (1996) Element-oriented and edge-oriented local error estimators for nonconforming finite element methods. ESAIM Math. Model. Numer. Anal., 30, 237-263.

John, V., Matthies, G., Schieweck, F. \& Tobiska, L. (1998) A streamline-diffusion method for nonconforming finite element approximations applied to convection-diffusion problems. Comput. Methods Appl. Mech. Eng., 166, 85-97.

Johnson, C., NÄVERT, U. \& Pitkëranta, J. (1984) Finite element methods for linear hyperbolic equations. Comput. Methods Appl. Mech. Eng., 45, 285-312. 
Johnson, C. \& Pitkëranta, J. (1986) An analysis of the discontinuous Galerkin method for a scalar hyperbolic equation. Math. Comput., 46, 1-26.

KARAKASHIAN, O. \& PASCAL, F. (2003) A posteriori error estimates for a discontinuous Galerkin approximation of second order elliptic problems. SIAM J. Numer. Anal., 41, 2374-2399.

Knobloch, P. \& Tobiska, L. (2003) The $P_{1}^{\text {mod }}$ element: a new nonconforming finite element for convectiondiffusion problems. SIAM J. Numer. Anal., 41, 436-456.

Matthies, G. \& Tobiska, L. (2001) The streamline-diffusion method for conforming and nonconforming finite elements of lowest order applied to convection-diffusion problems. Computing, 66, 343-364.

OUAzZI, A. \& TUREK, S. (2005) Unified edge-oriented stabilization of nonconforming finite element methods for incompressible flow problems. Ergebnisberichte des Instituts für Angewandte Mathematik, Nummer 284. FB Mathematik, Universität Dortmund.

SANGALLI, G. (2004a) Analysis of the advection-diffusion operator using fractional order norms. Numer. Math., 97, 779-796.

SANGALLI, G. (2004b) On robust a posteriori estimators for the advection-diffusion-reaction problem. Technical Report 04-55. Pavia, Italy: ICES - Instituto di Matematica Applicata e Tecnologie Informatiche del C.N.R.

STYNES, M. \& ToBiskA, L. (2001) The streamline-diffusion method for nonconforming $Q_{1}^{\text {rot }}$ elements on rectangular tensor-product meshes. IMA J. Numer. Anal., 21, 123-142.

VERfÜRTH, R. (1998) A posteriori error estimators for convection-diffusion equations. Numer. Math., 80, 641-663.

VERFÜRTH, R. (2005) Robust a posteriori error estimates for stationary convection-diffusion equations. SIAM J. Numer. Anal., 43, 1766-1782. 